\title{
Epífitas vasculares do Parque Nacional Serra de Itabaiana, Sergipe, Brasil
}

\author{
Kelianne Carolina Targino de Araújo * \\ Josuel Lima Santos \\ Juliano Ricardo Fabricante \\ Universidade Federal de Sergipe, Campus Universitário Professor Alberto Carvalho \\ Avenida Vereador Olímpio Grande, CEP 49510-200, Itabaiana - SE, Brasil \\ * Autor para correspondência \\ kelikarolina@hotmail.com
}

Submetido em 26/06/2018

Aceito em 28/01/2019

\section{Resumo}

O objetivo do presente trabalho foi realizar um levantamento florístico das epífitas vasculares do Parque Nacional Serra de Itabaiana, Sergipe. A falta de pesquisa sobre epifitismo no estado de Sergipe motivou o estudo no Parque Nacional Serra de Itabaiana (PARNASI), que detém uma grande importância para a conservação de espécies no estado. Diante disso, o trabalho buscou responder as seguintes perguntas: quantas e quem são as espécies de epífitas vasculares que ocorrem no PARNASI?; a quais categorias ecológicas as epífitas amostradas pertencem?; e qual a similaridade florística epifítica do PARNASI com outras áreas estudadas no Brasil? O inventário foi realizado através de caminhadas por todo o local (busca ativa), com coleta, herborização e acervo, no Herbário da Universidade Federal de Sergipe (ASE), de amostras de todas as espécies epífitas vasculares presentes no local. Foram coletadas 47 espécies distribuídas em 29 gêneros e 10 famílias. Os resultados da análise de similaridade demonstraram uma baixa semelhança florística entre a área de estudo e demais regiões brasileiras. O elevado número de táxons exclusivos nas áreas, estudadas e comparadas, revelam a grande importância de cada local para a conservação das espécies epífitas.

Palavra-chave: Epifitismo; Inventário florístico; Mata Atlântica

\section{Abstract}

Vascular epiphytes of Serra de Itabaiana National Park, Sergipe, Brazil. The objective of this work was to conduct a floristic survey of the vascular epiphytes of Serra de Itabaiana National Park (PARNASI) in Sergipe State. The lack of research about epiphytes in Sergipe was the reason for the study in PARNASI, which is very important for the conservation of species in the state. The study sought to answer three questions. How many and what are the species of vascular epiphytes that occur in PARNASI? What are the ecological categories of the epiphytes sampled? How similar is the epiphytic flora of PARNASI to other areas studied in Brazil? The inventory was carried out by walking throughout the park (active search). All vascular epiphytic species were collected, herborized and deposited in the UFS herbarium (ASE). A total of 47 species, in 29 genera and 10 families, were collected. The results of the similarity analysis showed low floristic similarity between the study area and those of other regions. The high number of exclusive taxa in the areas, studied and compared, reveals the great importance of each site for the conservation of epiphytic species.

Key words: Atlantic Forest; Epiphytic; Floristic inventory 


\section{Introdução}

O epifitísmo consiste na interação harmônica entre duas espécies, no qual uma planta (a epífita) se utiliza da outra (o forófito) como suporte (BENZING, 1995). As epífitas representam em torno de $10 \%$ de todas as espécies vasculares conhecidas, perfazendo aproximadamente 29.000 espécies (MADISON, 1977; KRESS, 1986; GENTRY; DODSON 1987). Embora não tenham contato com o solo, segundo Duarte e Gandolfi (2013), as epífitas adquirem recursos, para sua sobrevivência, por outros meios, como a atmosfera e pela lixiviação de compostos oriundos das copas das árvores.

Essas espécies têm a capacidade de influenciar de forma positiva os processos ecológicos e são fundamentais na manutenção dos ecossistemas, pois são capazes de fornecer distintos micro-habitat e microclimas, abrigando grande diversidade de vida (LUGO; SCATENA, 1992; KERSTEN; SILVA, 2001; DUARTE; GANDOLFI, 2013). As epífitas são fontes de recursos para os animais do dossel da floresta, além de local de nidificação, abrigo e reprodução (WAECHTER, 1992), contribuindo dessa forma para uma maior riqueza e diversidade faunística (NADKARNI, 1986; JOANITTI, 2013).

Além das importâncias citadas, as epífitas vasculares vêm sendo utilizadas como bioindicadoras de mudanças climáticas, poluição e danos ao ecossistema (LUGO; SCATENA, 1992), pois estudos realizados em várias partes do mundo têm demonstrado que a abundância, riqueza e estrutura das comunidades epífitas se modificam de acordo com o grau de interferência sobre as florestas tropicais (TURNER et al., 1994; HIETZ-SEIFERT et al., 1996; BARTHLOTT et al., 2001; NKONGMENECK et al., 2002). Apesar de sua grande importância para os ecossistemas florestais, os estudos sobre epifitísmo ainda são escassos no Brasil, mas, a despeito do aumento deles nas últimas décadas (FONTOURA et al., 1997; DISLICH; MONTAVANI, 1998; WAECHTER, 1998; DITTRICH et al., 1999; KERSTEN; SILVA, 2001; BORGO; SILVA, 2003; ROGALSKI; ZANIN, 2003; BATAGHIN et al., 2012), a maioria foi desenvolvido nas regiões Sul e Sudeste, sendo raros na região Nordeste, como o fizeram Dias-
Terceiro et al. (2014). Para o estado de Sergipe não há registro de nenhum trabalho científico voltado para esse grupo de plantas e, consequentemente, no Parque Nacional Serra de Itabaiana (PARNASI) não existem estudos específicos com as epífitas.

No parque podem ser observadas diferentes fitofisionomias, o que favorece a grande riqueza de espécies encontradas no seu interior. Em um levantamento florístico realizado por Mendes et al. (2010), foram registradas mais de 550 espécies. Conforme Carvalho e Villar (2005), existem, na área, 23 espécies de anfíbios, 32 de répteis, 123 de aves, 62 de mamíferos e 28 de abelhas.

Devido à importância do PARNASI para a conservação da biodiversidade brasileira, tendo em vista a falta de estudos sobre epífitas no estado, objetivou-se responder as seguintes perguntas: (i) quantas e quem são as espécies epifíticas vasculares que ocorrem no PARNASI; (ii) a quais categorias ecológicas pertencem as epífitas amostradas; (iii) qual a similaridade florística epifitica do PARNASI com outras áreas estudadas no Brasil? Sendo assim, esse trabalho teve como objetivo realizar um levantamento florístico das epífitas vasculares no Parque Nacional Serra de Itabaiana, Sergipe.

\section{Material e Métodos}

\section{Local de estudo}

O PARNASI $\left(10^{\circ} 25^{\prime} \mathrm{S}\right.$ e $\left.37^{\circ} 25^{\prime} \mathrm{W}\right)$ possui uma área de 7.966 ha com relevo levemente ondulado a ondulado, com altitudes que variam de 200 a 650 m (COSTA, 2014). Ele está situado em uma região de transição entre Floresta Ombrófila Aberta (Mata Atlântica) e Savana Estépica (Caatinga), abrigando muitas espécies importantes da fauna e flora brasileira (SOBRAL et al., 2007). O parque é composto predominantemente por Mata Atlântica, que se apresenta em diferentes condições de conservação. O clima regional é do tipo As', ou seja, segundo a classificação de Köeppen-Geiger, tropical com verão seco e moderado excedente hídrico no inverno, com uma umidade relativa que varia aproximadamente entre 58 a 94\%. Os solos variam bastante, podendo-se citar as Areias Quartzosas e os Litossolos, entre outros. 


\section{Método de amostragem}

O levantamento florístico foi realizado por meio de caminhadas por toda a área do PARNASI (busca ativa). Todos os indivíduos epifíticos que apresentavam material reprodutivo foram coletados, identificados e herborizados conforme procedimentos usuais propostos por Fidalgo e Bononi (1989). De 2015 a 2018 foram realizadas 30 expedições no local de estudo com duração média de $4 \mathrm{~h}$ cada. Todas as amostras foram depositadas no Herbário da Universidade Federal de Sergipe (ASE), São Cristóvão (SE). A classificação taxonômica foi elaborada de acordo com o Sistema APG III (2009) e a grafia dos nomes dos autores das espécies, segundo a Lista de Espécies da Flora do Brasil (2018).

As epífitas foram classificadas quanto a sua forma de vida em hemiepífitas primárias, hemiepífitas secundárias, holoepífitas obrigatórias e holoepífitas facultativas (BENZING, 1990), sendo: (i) holoepífitas obrigatórias: - aquelas plantas observadas vegetando apenas sobre outras plantas; (ii) holoepífitas facultativas: - aquelas plantas observadas vegetando tanto sobre outras plantas como no solo; (iii) hemiepífitas primárias: - aquelas plantas que vegetam inicialmente sobre outras plantas, mas que depois estabelecem contato com o solo; (vi) hemiepífitas secundárias: - aquelas plantas que inicialmente vegetam no solo, mas que depois crescem sobre outras plantas e perdem a ligação com o ambiente terrícola. As holoepífitas acidentais não foram consideradas no presente estudo, uma vez que são espécies essencialmente terrícolas e assim poderiam superestimar o número de espécies epifíticas.

Para avaliar a similaridade florística da área estudada com outras de regiões biogeográficas distintas (18 áreas) foi utilizado o coeficiente de Jaccard ( $\mathrm{Sj}$ ) (MÜLLER-DOMBOIS; ELLENBERG, 1974). A avaliação do grau de ajuste dos agrupamentos formados na análise foi verificada pelo coeficiente de correlação cofenética (SOKAL; ROHLF, 1962), e o método de agrupamento utilizado foi o Arithmetic Average Clustering (SNEATH; SOKAL, 1973). A análise foi realizada por meio do software MVSP $3.1^{\circ}(\mathrm{KOVACH}$, 2005).

\section{Resultados}

Foram amostradas 47 espécies epifíticas, distribuídas em 29 gêneros e 10 famílias (Tabela 1). A família Bromeliaceae foi a mais representativa, com dezoito espécies $(38,3 \%)$; seguida da família Orchidaceae, com doze espécies (25,5\%); Polypodeaceae,

TABELA 1: Lista de espécies epifíticas amostradas no Parque Nacional Serra de Itabaiana, Sergipe, suas respectivas categorias ecológicas e números de registro no Herbário da Universidade Federal de Sergipe (ASE) (№ Reg.). Sendo: $\mathrm{CE}=$ categoria ecológica; $\mathrm{HO}=$ holoepífita obrigatória; $\mathrm{HF}=$ holoepífita facultativa; $\mathrm{HP}=$ hemiepífita primária; HS = hemiepífita secundária.

\begin{tabular}{lllc}
\hline \multicolumn{1}{c}{ Família } & \multicolumn{1}{c}{ Espécie } & CE & № Reg. \\
\hline \multirow{2}{*}{ Araceae } & Anthurium affine Schott & HF & ASE38442 \\
& Anthurium scandens (Aubl.) Engl. & HO & $*$ \\
& Monstera adansonii Schott & HS & $*$ \\
& Philodendron acutatum Schott & HS & ASE38472 \\
& Philodendron fragrantissimum (Hook.) G.Don & HS & ASE37021 \\
\hline Bromeliaceae & Aechmea aquilega (Salisb.) Griseb. & HF & ASE37018 \\
& Aechmea lingulata (L.) Baker & HF & ASE37664 \\
& Aechmea marauensis Leme & HF & ASE37016 \\
& Aechmea mertensii (G. Mey.) Schult. \& Schult.f. & HF & ASE37017 \\
& Aechmea multiflora L.B.Sm. & HF & $*$ \\
& Aechmea nudicaulis (L.) Griseb & HF & ASE37662 \\
& Aechmea patentissima (Mart. ex Schult. \& Schult.f.) Baker & HF & ASE38466
\end{tabular}




\begin{tabular}{|c|c|c|c|}
\hline & Cryptanthus sp. & $\mathrm{HF}$ & $*$ \\
\hline & Cryptanthus sergipensis I. Ramírez & $\mathrm{HF}$ & ASE39076 \\
\hline & Guzmania lingulata (L.) Mez & $\mathrm{HF}$ & ASE37666 \\
\hline & Hohenbergia catingae Ule & $\mathrm{HF}$ & ASE37014 \\
\hline & Tillandsia bulbosa Hook.f. & $\mathrm{HO}$ & ASE37663 \\
\hline & Tillandsia gardneri Lindl & $\mathrm{HO}$ & ASE37019 \\
\hline & Tillandsia pohliana $\mathrm{Mez}$ & $\mathrm{HO}$ & ASE39086 \\
\hline & Tillandsia recurvata (L.) L. & $\mathrm{HO}$ & ASE39721 \\
\hline & Tillandsia stricta Sol. & $\mathrm{HO}$ & ASE39087 \\
\hline & Tillandsia usneoides (L.) L. & $\mathrm{HO}$ & ASE39866 \\
\hline & Vriesea simplex (Vell.) Berr & $\mathrm{HF}$ & ASE39080 \\
\hline \multirow[t]{2}{*}{ Cactaceae } & Epiphyllum phyllanthus (L.) Haw. & $\mathrm{HO}$ & ASE39090 \\
\hline & Rhipsalis lindbergiana K.Schum. & $\mathrm{HO}$ & ASE37661 \\
\hline Cyatheaceae & Cyathea phalerata Mart. & $\mathrm{HF}$ & ASE38477 \\
\hline Gesneriaceae & Codonanthe mattos-silvae Chautems & $\mathrm{HO}$ & ASE38481 \\
\hline Lygodiaceae & Lygodium venustum $\mathrm{Sw}$. & $\mathrm{HO}$ & ASE38473 \\
\hline \multirow[t]{12}{*}{ Orchidaceae } & Alatiglossum sp. & $\mathrm{HO}$ & $*$ \\
\hline & Campylocentrum crassirhizum Hoehne & $\mathrm{HO}$ & ASE38482 \\
\hline & Catasetum uncatum Rolfe & $\mathrm{HO}$ & $*$ \\
\hline & Epidendrum sp. & $\mathrm{HO}$ & ASE38468 \\
\hline & Epidendrum corpophorum Barb. Rodr. & $\mathrm{HO}$ & ASE37665 \\
\hline & Jacquiniella globosa (Jacq.) Schltr. & $\mathrm{HO}$ & ASE39078 \\
\hline & Polystachya concreta (Jacq.) Garay \& Sweet & $\mathrm{HO}$ & ASE38471 \\
\hline & Polystachya estrellensis Rchb.f. & $\mathrm{HO}$ & ASE37023 \\
\hline & Scaphyglottis sp. & $\mathrm{HO}$ & ASE38475 \\
\hline & Scaphyglottis fusiformis (Griseb) R.E. Schultes & $\mathrm{HO}$ & $*$ \\
\hline & Trichocentrum fuscum Lindl. & $\mathrm{HO}$ & * \\
\hline & Vanilla palmarum (Salzm. ex Lindl.) Lindl. & HS & ASE39081 \\
\hline \multirow[t]{3}{*}{ Piperacae } & Peperomia circinnata Link & $\mathrm{HO}$ & $*$ \\
\hline & Peperomia obtusifolia (L.) A.Dietr. & HS & $*$ \\
\hline & Peperomia glabella (Sw.) A.Dietr. & $\mathrm{HO}$ & ASE39876 \\
\hline \multirow[t]{6}{*}{ Polypodiaceae } & Campyloneurum nitidum (Kaulf.) C.Presl & HF & ASE39088 \\
\hline & Microgramma vacciniifolia (Langsd. \& Fisch.) Copel. & $\mathrm{HO}$ & ASE38478 \\
\hline & Microgramma squamulosa (Kaulf.) de la Sota & $\mathrm{HO}$ & ASE39736 \\
\hline & Pleopeltis astrolepis (Liebm.) E.Fourn. & $\mathrm{HO}$ & ASE39075 \\
\hline & Pleopeltis macrocarpa (Bory ex Willd.) Kaulf. & $\mathrm{HO}$ & ASE39082 \\
\hline & Serpocaulon triseriale (Sw.) A.R.Sm. & $\mathrm{HO}$ & ASE38467 \\
\hline Pteridaceae & Vittaria lineata (L.) Sm. & $\mathrm{HO}$ & * \\
\hline
\end{tabular}

*Espécies coletadas em processo de tombo no Herbário ASE.

com sete espécies (14,9\%); Araceae, com cinco espécies $(10,63 \%)$; Piperaceae, com três $(6,38 \%)$; e Cactaceae com duas espécies (4,25\%). As demais famílias, Cyathaceae, Gesneriaceae, Lygodiaceae e Pteridaceae, apresentaram apenas uma espécie cada (2,12\%). Em relação às categorias ecológicas, 31 (65,9\%) espécies foram classificadas como holoepífitas obrigatórias, 15 (31,9\%) como holoepífitas facultativas, e cinco (10,6\%) como hemiepífitas secundárias. Nenhuma hemiepífita primária foi observada na área de estudo. 
Em relação à similaridade, todos os trabalhos comparados apresentaram uma baixa semelhança específica entre si (Figura 1). Os maiores índices encontrados entre a área de estudo e os demais trabalhos consultados foram de 9,1\%, na Floresta Estacional Semidecidual, Serra da Babilônia, em Minas Gerais (BARBOZA et al., 2015 ) e de 8,1\%, no Cerrado, Estação Ecológica de Jataí, em São Paulo.

\section{Discussão}

Em trabalhos realizados na região Nordeste, como o de Castro et al. (2016), em uma área de Caatinga, município de Buíque (PE), o número de espécies foi menor quando comparado ao presente estudo, tendo os autores encontrado 16 espécies na área de amostragem, e, entre elas, apenas quatro similares [Philodendrum acutatum Schott.; Vanilla palmarum (Salzm. ex Lindl.) Lindl.; Serpocaulon triseriale (Sw.) A.R.Sm.; Tillandsia recurvata (L.) L.]. Também foi realizada a mesma observação em relação aos resultados obtidos por Dias-Terceiro et al. (2014), em uma área de Floresta Ombrófila Aberta, município de Areia (PB), com o total de 12 espécies, sendo que somente três também estiveram presentes no PARNASI [Polystachya concreta (Jacq.) Garay \& H.R. Sweet; Microgramma vacciniifolia (Langsd. \& Fisch.) Copel. e Pleopeltis macrocarpa (Bory ex Willd.) Kaulf.]. É importante frisar que as áreas

FIGURA 1: Cluster produzido pela análise de similaridade de Jaccard. Sendo: 1 - área estudada; 2 - área de Floresta Estacional Semidecidual, Maringá (PR) (DETTKE et al., 2008); 3 - área de Floresta Ombrófila Mista e Floresta Estacional Decidual, Palmeira das Missões (RS) (ALVES et al., 2014); 4 - área de Floresta Ombrófila Mista e Floresta Estacional Semidecidual, Mourão (RS) (GERALDINO et al., 2010); 5 - área de Mata Paludosa do Faxinal, Torres (RS) (WAECHTER, 1986); 6 - área de Campos, Eldorado do Sul (RS) (GIONGO; WAECHTER, 2004); 7 - área de Floresta Ombrófila Mista Altomontana, Guarapuava e Pinhão (PR) (KERSTEN, 2006); 8 - área de Cerrado, Jataí (SP) (BATAGHIN et al., 2012); 9 - área de Floresta Estacional Semidecidual, Borebi (SP) (FABRICANTE et al., 2007); 10 - área de Floresta Estacional Semidecidual, Ouro Preto (MG) (FERREIRA, 2011); 11 - área de Floresta Ombrófila Densa, Serra da Mantiqueira (MG) (ALVES; MENININETO, 2014); 12 - área de Floresta Sazonal Semidecidual, Serra da Babilônia (MG) (BARBOZA et al., 2015 ); 13 - área de Floresta Ombrófila Aberta, Areia (PB) (DIAS-TERCEIRO et al., 2014); 14 - área de Floresta Estacional Semidecidual, João Pessoa (PB) (GADELHA NETO; BARBOSA, 2012); 15 - área de Caatinga, Buíque (PE) (CASTRO et al., 2016 ); 16 - área de Floresta Ombrófila Densa, Manaus (AM) (SILVA, 2012); 17 - área da Amazônia, Maracanã (PA) (QUARESMA; JARDIM, 2014) ; 18 - área da Amazônia, Amazonas (AM) (BOELTER et al., 2014); 19 - área de Floresta Ombrófila Aberta, Acrelândia (AC) (OBERMÜLLER et al., 2012).

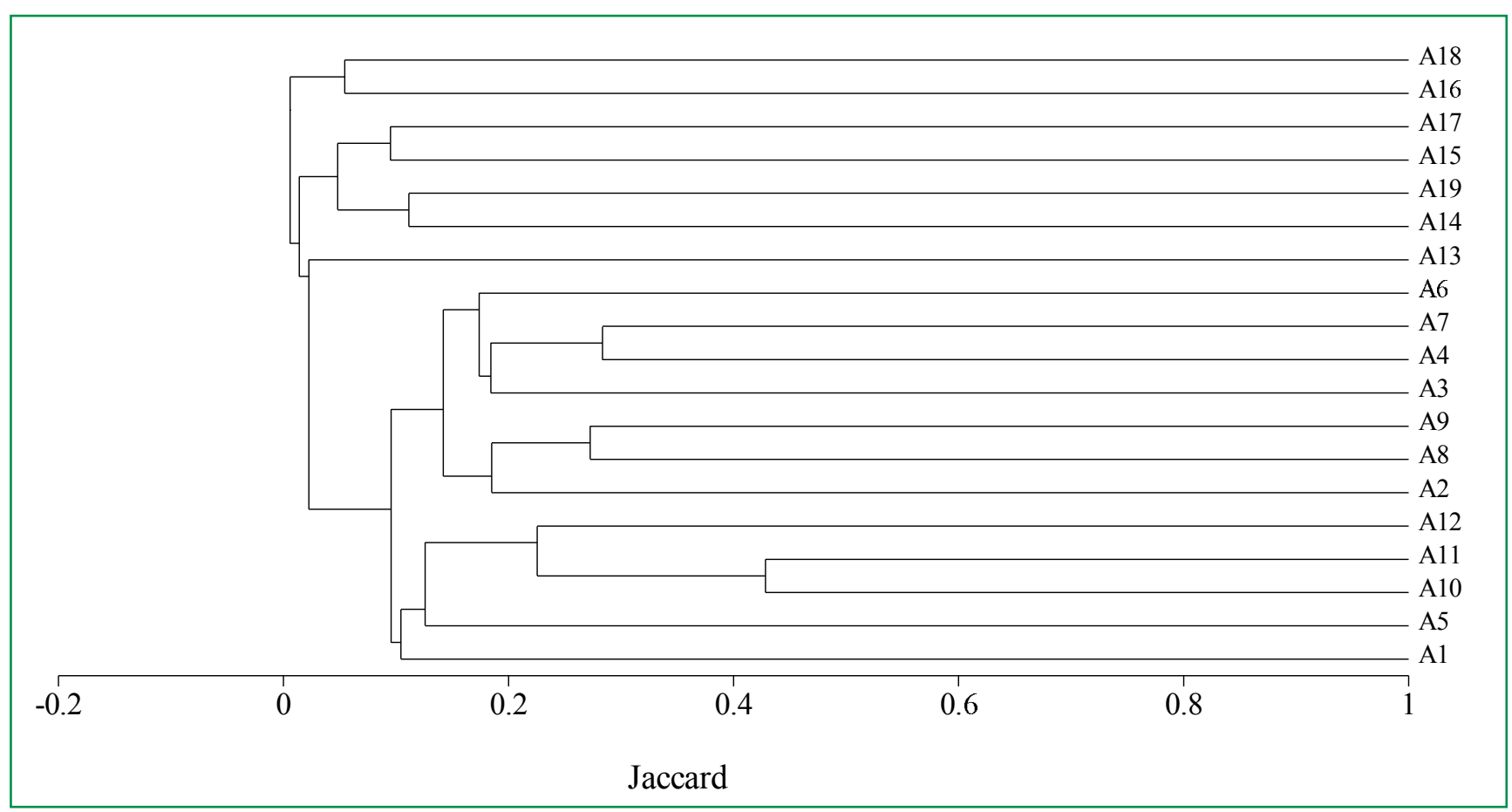


possuem discrepâncias nas suas dimensões, fator esse que pode ser o responsável pelas diferenças observadas.

O número de espécies epífitas amostradas também foi maior quando comparado a estudos desenvolvidos em outras regiões do país (GIONGO; WAECHTER, 2004; DETTKE et al., 2008; BATAGHIN et al., 2012; DUARTE; GANDOLFI, 2013; ALVES et al., 2014;), reforçando a necessidade e a importância de se intensificarem os estudos com epífitas vasculares no Nordeste brasileiro.

Quanto às famílias mais representativas, Bromeliaceae e Orchidaceae foram as mais encontradas. Outros estudos obtiveram resultados semelhantes aos obtidos no PARNASI, a exemplo do levantamento realizado por Perleberg et al. (2013), em uma área de Floresta Estacional Semidecidual, no município de Pelotas (RS); de Fontoura et al. (1997) e de Kersten e Silva (2001), em áreas de Floresta Ombrófila Densa, no Rio de Janeiro e no Paraná, respectivamente; de Leitman et al., (2014), no Parque Nacional Serra das Lontras (BA), em região de Mata Atlântica; e de Gonçalves e Waechter (2003), no norte da planície costeira do Rio Grande do Sul. Bromeliaceae e Orchidaceae apresentam espécies com diversas adaptações para sobreviverem ao estresse hídrico (STAUDT et al., 2012). Muitas bromélias, por exemplo, possuem tricomas nas folhas ou tanques para captação e armazenamento de água (BENZING; SHEEMAN, 1978). Muitas espécies de orquídeas, por sua vez, apresentam pseudobulbos que permitem o armazenamento de água e auxiliam na manutenção do balanço hídrico (BRAGA, 1977). Certamente, esses atributos capacitam essas famílias a se manter na condição de epífita, sobre os ramos e troncos de outras espécies, onde a intermitência de água é um fator limitante, justificando sua representatividade nos diferentes estudos destacados acima.

As bromélias também desempenham um importante papel ecofisiológico nas florestas, pois estas constituem micro-habitat para diversos animais, entre eles, insetos, anfíbios e répteis (MOREIRA et al., 2006). Quanto à família Orchidaceae, são fonte de recursos alimentícios para vários grupos de insetos (Hymenoptera, Diptera, Lepidoptera e Coleoptera) e também para aves (VAN-DER-PIJL; DODSON,
1966). Algumas espécies de bromélias e de orquídeas ainda são excelentes bioindicadoras ambientais, pois são sensíveis às interferências antrópicas (ZOTZ; ANDRADE, 2002). Um ponto importante que deve ser levado em consideração é a diminuição no número de epífitas em florestas secundárias. Embora muitas delas, como as bromélias e orquídeas, tenham diversos meios de adaptação, estudos realizados por Nardkarni et al. (2004) afirmam que florestas secundárias apresentam apenas uma fração da biomassa das epífitas presentes nas florestas primárias. Muito provavelmente, a diminuição no número de epífitas está relacionada com a fragmentação florestal, uma atividade que traz, como efeito, o isolamento de populações, dificultando a colonização das epífitas em novas áreas (CAIN et al., 2000; GARCIA et al., 2011).

Em outros trabalhos também foram observados resultados semelhantes quanto à categoria ecológica, com mais da metade das espécies pertencentes às holoepífitas, a exemplo dos trabalhos de Kersten (2006), de Mania e Monteiro (2010) e de Alves et al. (2014), tendo Dittrich et al. (1999), Kersten e Silva (2001), Borgo et al. (2002) e Borgo e Silva (2003) concluído ser essa a categoria ecológica mais comum para a flora do Brasil.

Os resultados da análise de similaridade florística demonstraram haver uma baixa semelhança específica entre a área de estudo e as de outras regiões brasileiras, ou mesmo entre as áreas estudadas na região Nordeste (Figura 1). As maiores similaridades foram observadas no trabalho realizado por Barboza et al. (2015), em uma área de Floresta Sazonal Semidecidual de Minas Gerais, com treze espécies comuns entre os estudos [Anthurium scandens (Aubl.) Engl.; Monstera adansonii Schott; Aechmea nudicaulis (L.) Griseb; Tillandsia recurvata; Tillandsia stricta Sol.; Tillandsia usneoides (L.) L.; Rhipsalis lindbergiana K.Schum.; Campylocentrum crassirhizum Hoehne; Polystachya estrellensis Rchb.f.; Peperomia glabella (Sw.) A.Dietr.; Campyloneurum nitidum (Kaulf.) C.Presl; Microgramma squamulosa (Kaulf.) de la Sota e Pleopeltis astrolepis (Liebm.) E. Fourn.], e por Bataghin et al. (2012), em uma área de Cerrado (Campo sujo, Campo limpo, Cerrado stricto senso e Cerradão), na estação ecológica de Jataí, 
SP, onde oito espécies foram similares [Aechmea nudicaulis (L.) Griseb; Tillandsia recurvata; Tillandsia stricta; Epiphyllum phyllanthus (L.) Haw.; Polystachya estrellensis Rchb.f.; Peperomia glabella (Sw.) A. Dietr.; Campyloneurum nitidum (Kaulf.) C.Presl e Microgramma squamulosa (Kaulf.) de la Sota].

A baixa similaridade observada aponta para o elevado número de táxons exclusivos nas áreas, estudadas e comparadas, revelando assim a grande importância de cada local para a conservação das espécies epífitas vasculares brasileiras.

\section{Referências}

ALVES, F. E.; MENINI NETO, L. Vascular epiphytes in a forest fragment of Serra da Mantiqueira and floristic relationships with Atlantic high altitude areas in Minas Gerais. Brazilian Journal of Botany, São Paulo, v. 37, p. 187-196, 2014.

ALVES, M. E. O.; BRUN, C.; FORNO, R. S. D.; ESSI, L. Levantamento de espécies epífitas vasculares da zona urbana do município de Palmeira das Missões, RS, Brasil. Ciência e Natura, Santa Maria, v. 36, n. 3, p. 268-276, 2014.

APG III - ANGIOSPERM PHYLOGENY GROUP. An update of the Angiosperm Phylogeny Group classification for the orders and families of flowering plants: APG III. Botanical Journal of the Linnean Society, London, v. 161, p. 105-121, 2009.

BARBOZA, D. E. F.; BASÍLIO, G. A.; SILVA, F. R.; NETOMENINE, L. Vascular epiphytes in a remnant of seasonal semideciduous forest in the zona da mata, state of Minas Gerais, Brazil. Bioscience Journal, Uberlândia, v. 31, n. 2, p. 623-633, 2015. BARTHLOTT, W.; SCHMIT-NEUERBURG, V.; NIEDER, J.; ENGWALD, S. Diversity and abundance of vascular epiphytes: a comparison of secondary vegetation and primary montane rain forest in the Venezuelan Andes. Plant Ecology, Dordrecht, v. 152, p. 145-156, 2001.

BATAGHIN, F. A.; MULlER, A.; PIRES, J. S. R.; BARROS, A. T. F.; SCARIOT, E. C. Riqueza e estratificação vertical de epífitas vasculares na Estação ecológica de Jataí - área do Cerrado no Sudeste do Brasil. Hoehnea, São Paulo, v. 39, n. 4, p. 615-626, 2012.

BENZING, D. H. Vascular epiphytes: general biology and related biota. Cambridge: University Press Cambridge, 1990. 346 p.

BENZING, D. H. The physical mosaic and plant variety in forest canopies. Selbyana, Sarasota, v. 16, n. 2 p. 159-168, 1995.

BENZING, D. H.; SHEEMANN, J. Nutritional piracy and host decline: a new perspective on the epiphyte-host relationship. Selbyana, Sarasota, v. 2, n. 2, p. 133-148, 1978.

BOELTER, C. R.; DAMBROS, C. S.; NASCIMENTO, H. E. M.; ZARTMAN, C. E. A tangled web in tropical tree-tops: effects of edaphic variation, neighborhood phorophyte composition and bark characteristics on epiphytes in a central Amazonian forest. Journal of Vegetation Science, Washington, v. 25, p. 1090-1099, 2014.
BORGO, M.; SILVA, S. M. Epífitas vasculares em fragmentos de Floresta Ombrófila Mista, Curitiba, Paraná, Brasil. Revista Brasileira de Botânica, São Paulo, v. 26, n. 3, p. 391-401, 2003.

BORGO, M.; SILVA, S. M.; PETEAN, M. Epífitos vasculares em um remanescente de Floresta Estacional Semidecidual, município de Fênix, PR, Brasil. Acta Biologica Leopoldensia, São Leopoldo, v. 24, n. 2, p. 121-130, 2002.

BRAGA, P. I. S. Aspectos ecofisiológicos de Orchidaceae da Amazonia II. Anatomia ecológica foliar de espécies com metabolismo CAM de uma campina da amazonia central. Acta Amazonica, Manaus, v. 7, n. 2, p. 1-89, 1977.

CAIN, M. L.; MILLIGAN, B. G.; STRAND, A. E. Longdistance seed dispersal in plant populations. American Journal of Botany, St. Louis, v. 87, p. 1217-1227, 2000.

CARVAlHO, M.; VILAR, J. C. Parque Nacional Serra de Itabaiana: levantamento da biota. Aracaju: Ibama, 2005. 257 p.

CASTRO, R. A.; FABRICANTE, J. R.; FILHO, J. A. S. Palmeira Syagrus Coronata(Mart.) Beec. Para a Conservação da Riqueza e Diversidade de Epécies Epífitas Vasculares na Caatinga. Revista Árvore, Viçosa, v. 40, n. 1, p. 1-12, 2016.

COSTA, C. C. Parque Nacional Serra de Itabaiana - SE: realidade e gestão. Revista Monografias Ambientais, Santa Maria, v. 13, n. 5, p. 3933-3951, 2014.

DETTKE, G. A.; ORFRINIZ, A. C.; GUTIERREZ, M. A. M. Composição florística e distribuição de epífitas vasculares em remanescente alterado de Floresta Estacional Semidecidual no Paraná, Brasil. Rodriguésia, Rio de Janeiro, v. 59, n. 4, p. 859-872, 2008.

DIAS-TERCEIRO, R. G.; GOMES, V. S.; PEIXOTO, G. M.; MENEZES, M. C.; FABRICANTE, J. R.; ALBUQUERQUE, M. B. Distribuição horizontal de epífitas vasculares em um fragmento de Floresta Ombrófila Aberta no Nordeste brasileiro. Natureza Online, Santa Teresa, v. 12, n. 4, p. 195-200, 2014.

DISLICH, R.; MANTOVANI, W. A flora de epífitas vasculares da Reserva da Cidade Universitária "Armando de Salles Oliveira" (São Paulo, Brasil). Boletim de Botânica, São Paulo, v. 17, p. 6183, 1998.

DITTRICH, V. A. O.; KOZERA, C.; SILVA, S. M. Levantamento florístico de epífitos vasculares no Parque Barigüi, Paraná, Brasil. Iheringia (Série Botânica), Porto Alegre, v. 52, p. 11- 22, 1999.

DUARTE, M. M.; GANDOLFI, S. Enriquecimento de florestas em processo de estauração: aspectos de epífitas e forófitos que podem ser considerados. Hoehnea, São Paulo, v. 40, n. 3, p. $507-$ 514, 2013.

FABRICANTE， J. R.; SANTOS, F. S.; OLIVEIRA， I. G.; FERREIRA, J. C.; MICHELIN, A. C. Caracterização florística do componente epifítico vascular ocorrente nas formações florestais da fazenda noiva de colina, município de Borebi (SP). Salusvita, Bauru, v. 26, n. 2, p. 7-21, 2007.

FERREIRA, M. T. M. Composição florística e distribuição vertical de epífitas vasculares sobre indivíduos de Guapira opposita (Vell.) Reitz (Nyctaginaceae) em um fragmento florestal na Serra da Brígida, Ouro Preto, MG. 2011. 69 f. Dissertação (Mestrado em Ecologia de Biomas Tropicais) - Universidade Federal de Ouro Preto, Ouro Preto. 2011. 
FIDALGO, O.; BONONI, V. L. R. Técnicas de coleta, preservação e herborização de material botânico. In: FIDALGO, O.; BONONI, V. L. R. (Ed.). Técnicas de coleta, preservação e herborização de material botânico. 4. ed. São Paulo: Instituto de Botânica, 1984. $61 \mathrm{p}$.

FONTOURA, T.; SYLVESTRE, L. S.; VAZ, A. M. S.; VIEIRA, C. M. Epífitas vasculares, hemiepífitas e hemiparasitas da Reserva Ecológica de Macaé de Cima. In: LIMA, H. C.; GUEDES-BRUNI, R. R. (Ed.). Serra de Macaé de Cima: diversidade florística e conservação da Mata Atlântica. 1 ed. Rio de Janeiro: Jardim Botânicoo, 1997. p. 89-102.

GADELHA NETO, P. da COSTA; BARBOSA, M. R. de V. Angiospermas trepadeiras, epífitas e parasitas da Mata Do Buraquinho, João Pessoa, Paraíba. Revista Nordestina de Biologia, João Pessoa, v. 21, n. 1, p. 81-92, 2012.

GARCIA, L. C.; HOBBS, R. J.; RIBEIRO, D. B.; TAMASHIRO, J. Y.; SANTOS, F. A. M.; RODRIGUES, R. R. Changes in vegetation along restoration time: influence of composition and diversity of planted trees on natural regeneration. In: World CONFERENCE ON ECOLOGICAL RESTORATION, 4, 2011, Mérida. Abstracts... Mérida: Society for Ecological Restoration International, 2011. p. 81-82.

GENTRY, A. H.; DODSON, C. H. Contribution of nontrees to species richness of a tropical rainforest. Biotropica, Malden, v. 19, n. 2, p. $149-156,1987$.

GERAldinO, H. C. L.; CAXAMBÚ, M. G.; SOUZA, D. C. Composição florística e estrutura da comunidade de epífitas vasculares em uma área de ecótono em Campo Mourão, PR, Brasil. Acta Botanica Brasilica, Belo Horizonte, v. 24, n. 2, p. 469-482, 2010 .

GIONGO, C.; WAECHTER, J. L. Composição florística e estrutura comunitária de epífitos vasculares em uma floresta de galeria na Depressão Central do Rio Grande do Sul. Revista Brasileira de Botânica, São Paulo, v. 27, n. 3, p. 563-572, 2004

GONÇALVES, C. N.; WAECHTER, J. L. Aspectos florísticos e ecológicos de epífitos vasculares sobre figueiras isoladas no norte da planície costeira do Rio Grande do Sul. Acta Botanica Brasilica, Belo Horizonte, v. 17, n. 1, p. 89-100, 2003.

HIETZ-SEIFERT, U.; HIETZ, P.; GUEVARA, S. Epiphyte vegetation and diversity on remmant trees after forest clearance in southern Veracruz. Biological Conservation, Boston, v. 75, p. $103-$ 111,1996

JOANITTI, S.A. Epifitismo vascular em três formações vegetais distinta: Mata de brejo, Floresta Estacional Semidecidual e Cerradão, pertencentes ao município de Bauru, estado de São Paulo. 2013. 66 f. Dissertação (Mestrado em Ciências Biológicas Botânica) - Universidade Estadual Paulista, Botucatu. 2013.

KERSTEN, R. A. Composição florística de epífitos vasculares na Bacia de Iguaçu. 2006. 218 f. Tese (Doutorado em Engenharia Florestal) - Universidade Federal do Paraná, Curitiba. 2006.

KERSTEN, R. A.; SILVA, S. M. Epífitas vasculares em planície litorânea no Paraná, Brasil. Revista Brasileira de Botânica, São Paulo, v. 24, n. 2, p. 213-226, 2001.

KOVACH, W. L. MVSP - A multivariate statistical package for windows, ver. 3.1. Pentraeth: Kovach Computing Services, 2005. $133 \mathrm{p}$.
KRESS, J. W. The systematic distribution of vascular epiphytes: an update. Selbyana, Sarasota, v. 9, n. 1, p. 2-22, 1986.

LEITMAN, P.; AMORIM, A.; NETO, L. M.; FORZZA, R. C. Epiphytic angiosperms in a mountain forest in southern Bahia, Brazil. Biota Neotropica, Campinas, v. 14, n. 2, p. 1-12, 2014.

LISTA DE ESPÉCIES DA FLORA DO BRASIL. Jardim Botânico do Rio de Janeiro, Rio de Janeiro. Disponível em <http:// floradobrasil.jbrj.gov.br>.Acesso em: 26 novembro de 2018.

LUGO, A. E.; SCARTENA, F. N. Epiphytes and climate change research in the Caribbean: a proposal. Selbyana, Sarasota, v. 13, p. 123-130, 1992.

MADISON, M. Vascular epiphytes: their systematic occurrence and saliente features. Selbyana, Sarasota, v. 5, n. 2, p. 207-213, 1977.

MANIA, L. F.; MONTEIRO, R. Florística e ecologia de epífitas vasculares em um fragmento de floresta de restinga, Ubatuba, SP, Brasil. Rodriguésia, Rio de Janeiro, v. 61, n. 4, p. 705-713, 2010.

MENDES, K.; GOMES, P.; ALVES, M. Floristic inventory of a zone of ecological tension in the Atlantic Forest of Northeastern Brazil. Rodriguésia, Rio de Janeiro, v. 61, n. 4, p. 669-676, 2010.

MOREIRA, B. A.; WANDERLEY, M. G.; BARROS, M. A. V. C. Bromélias: importância ecológica e diversidade. Taxonomia e morfologia - Curso de Capacitação de Monitores. São Paulo: Instituto de Botânica, 2006. 12 p.

MÜLLER-DOMBOIS, D.; ELLENBERG, H. Aims and methods of vegetation ecology. New York: John Wiley e Sons, 1974. 547 p.

NADKARNI, N. M. An ecological overview and checklistof vascular epiphytes in the Monteverde cloud forestreserve, Costa Rica. Brenesia, San Jose, v. 24, p. 55-632, 1986.

NADKARNI, N. M.; PARKER, G. G.; RINKER, H. B.; JARZEN, D. M. The nature of forest canopies. In: LOWMAN, M. D.; RINKER, H. B. (Ed.). Forest canopies. Burlington: Elsevier, 2004. p. 3-23.

NKONGMENECK, B. A.; LOWMAN, M. D.; ATWOOD, J. T. Epiphyte diversity in primary and fragmented forests of Cameroon, Central Africa: a preliminar survey. Selbyana, Sarasota, v. 23, n. 1, p. 121-130, 2002.

OBERMÜLLER, F. A.; SILVEIRA, M.; SALIMON, C. I.; DALY, D. C. Epiphytic (including hemiepiphytes) diversity in three timber species in the southwestern Amazon, Brazil. Biodiversity and Conservation, New York, v. 21, p. 565-575, 2012

PERLEBERG, T. D.; GARCIA, E. N.; PITREZ, S. R. Epífitos vasculares em área com Floresta Estacional Semidecidual, Pelotas, Rio Grande do Sul, Brasil. Ciência e Natura, Santa Maria, v. 35, n. 2 , p. $65-73,2013$.

QUARESMA, A. C.; JARDIM, M. A. G. Floristic composition and spatial distribution of vascular epiphytes in the restingas of Maracanã, Brazil. Acta Botanica Brasilica, Belo Horizonte, v. 28, n. 1, p. 68-75, 2014.

ROGALSKI, J. M.; ZANIN, E. M. Composição florística de epífitas vasculares no Estreito de Augusto Cézar, Floresta Estacional Decidual do Rio Uruguai, RS, Brasil, Revista Brasileira de Botânica, São Paulo, v. 26, n. 4, p. 551-556, 2003.

SILVA, J. J. V. Distribuição espacial de epífitas vasculares na Amazônia Central. 2012. 67 f. Dissertação (Mestrado em 
Diversidade Biológica) - Universidade Federal do Amazonas, Manaus. 2012.

SNEATH, P. H. A.; SOKAL, R. R. Numerical taxonomy: the principles and practice of numerical classification. San Francisco: Freeman, 1973. $573 \mathrm{p}$

SOBRAL, I. S.; SANTANA, R. K. O.; GOMES, L. J.; COSTA, M.; RIBEIRO, G. T.; SANTOS, J. R. Avaliação dos impactos ambientais no Parque Nacional Serra de Itabaiana - SE. Caminhos de Geografia, Uberlândia, v. 8, n. 24, p. 102-110, 2007.

SOKAL, R. R.; ROHLF, F. J. The comparison of dendrograms by objective methods. Táxon, Berlin, v. 11, n. 1, p. 30-40, 1962.

STAUDT, M. G.; LIPPERT, A. P. U.; CUNHA, S.; BECKER, D. F. P.; MARCHIORETTO, M. S.; SCHMIT, T. Composição florística de epífitos vasculares do Parque Natural Municipal Tupancy, Arroio do Sal, RS - Brasil. Instituto Anchietano de Pesquisas, São Leopoldo, v. 1, n. 63, p. 177-188, 2012.

TURNER, I. M.; TAN, H. T. W.; WEE, Y. C.; IBRAHIN, A. B.; CHEW, P. T.; CORLETT, R. T. A study of plant species extinction in Singapore: lessons for the conservation of tropical biodiversity. Conservation Biology, San Francisco, v. 8, n. 3, p. 705-712, 1994.
VAN-DER-PIJL, L.; DODSON, C. H. Orchid flowers: their pollination and evolution. University of Miami Press, Coral Gables, 1966. 214 p.

WAECHTER, J. L. Epífitos vasculares da mata paludosa do Faxinal, Torres, Rio Grande do Sul, Brasil. Iheringia Série Botânica, Porto Alegre, v. 34, n. 1, p. 39-49, 1986.

WAECHTER, J. L. O epifitismo vascular na Planície Costeira do Rio Grande do Sul. 1992. 163 f. Tese (Doutorado em Ecologia e Recursos Naturais) - Universidade Federal de São Carlos, São Carlos. 1992.

WAECHTER, J. L. Epifitismo vascular em uma Floresta de Restinga do Brasil Subtropical. município de Santa Maria, Rio Grande do Sul. Ciência e Natura, Santa Maria, v. 20, n. 1, p. 43-66, 1998.

ZOTZ, G.; ANDRADE, J. L. La ecología y la fisiología de las epifitas y las hemiepífitas. In: GUARIGUATA, M.; CATAN, G. (Ed.). Ecología y conservación de bosques neotropicales, San José. 1. ed. San José: Editorial Libro Universitario Regional, 2002. p. 271-296. 